\title{
Topological phases in ultracold polar-molecule quantum magnets
}

\author{
Salvatore R. Manmana,,${ }^{1,2,3}$ E. M. Stoudenmire, ${ }^{4}$ Kaden R. A. Hazzard, ${ }^{2,3}$ Ana Maria Rey, ${ }^{2}$ and Alexey V. Gorshkov ${ }^{5}$ \\ ${ }^{1}$ Institute for Theoretical Physics, University of Göttingen, D-37077 Göttingen, Germany \\ ${ }^{2}$ JILA, NIST and Department of Physics, University of Colorado, Boulder, Colorado 80309, USA \\ ${ }^{3}$ Kavli Institute for Theoretical Physics, University of California, Santa Barbara, California 93106, USA \\ ${ }^{4}$ Department of Physics and Astronomy, University of California, Irvine, California 92697, USA \\ ${ }^{5}$ Institute for Quantum Information and Matter, California Institute of Technology, Pasadena, California 91125, USA
}

(Received 19 October 2012; published 26 February 2013)

\begin{abstract}
We show how to use polar molecules in an optical lattice to engineer quantum spin models with arbitrary spin $S \geqslant 1 / 2$ and with interactions featuring a direction-dependent spin anisotropy. This is achieved by encoding the effective spin degrees of freedom in microwave-dressed rotational states of the molecules and by coupling the spins through dipolar interactions. We demonstrate how one of the experimentally most accessible anisotropies stabilizes symmetry protected topological phases in spin ladders. Using the numerically exact density matrix renormalization group method, we find that these interacting phases-previously studied only in the nearestneighbor case-survive in the presence of long-range dipolar interactions. We also show how to use our approach to realize the bilinear-biquadratic spin-1 and the Kitaev honeycomb models. Experimental detection schemes and imperfections are discussed.
\end{abstract}

DOI: 10.1103/PhysRevB.87.081106

PACS number(s): 67.85.-d, 33.80.-b, 75.10.Jm, 75.10.Pq

Recent advances in ultracold polar molecules, ${ }^{1-3}$ Rydberg atoms, ${ }^{4,5}$ magnetic atoms,,${ }^{6,7}$ and magnetic defects in solids ${ }^{8-10}$ have spurred tremendous interest in exotic strongly correlated many-body phenomena arising from anisotropic, long-ranged dipole-dipole interactions. ${ }^{11-46}$ The types of anisotropies realizable with these interactions are typically limited to simple changes of the interaction sign and magnitude according to the spherical harmonic $Y_{2,0} \propto 1-3 \cos ^{2} \theta$, where $(\theta, \phi)$ are the spherical coordinates of the vector connecting the two interacting dipoles. ${ }^{11,13,32,33}$

In this Rapid Communication we show, in the context of polar molecules, that microwave dressing provides a tremendous degree of simultaneous control over five independent dipoledipole interaction terms whose angular dependences are given by the rank-2 spherical harmonics. This opens the door to simulating well-known models including the spin-1/2 XXZ model with a direction-dependent spin anisotropy, the spin1 bilinear-biquadratic model, ${ }^{47}$ and the Kitaev honeycomb model. ${ }^{48}$ Thanks to the use of direct dipole-dipole coupling, the resulting interactions are stronger and hence easier to observe experimentally than other-potentially direction-dependentspin-spin interactions such as superexchange in ultracold atoms $^{49}$ or perturbative dipole-dipole-mediated couplings between polar molecules. ${ }^{16,17}$

As a specific example demonstrating the reach of our method, we show how to design a spin- $1 / 2 X X Z$ model with direction-dependent spin anisotropy using a minimal and experimentally reasonable microwave configuration. In a two-legged ladder geometry with nearest-neighbor interactions, this model has been shown to exhibit symmetry protected topological (SPT) phases. ${ }^{50}$ These phases are exotic gapped states of matter distinct from trivial gapped phases when specific symmetries are present. They have recently attracted extensive interest ${ }^{51-59}$ because they do not fit within the framework of Landau symmetry breaking and possess exotic properties such as topologically protected edge states, ${ }^{60}$ nonlocal order parameters, ${ }^{61,62}$ and unique entanglement properties. ${ }^{57,63}$ Using the density matrix renormalization group

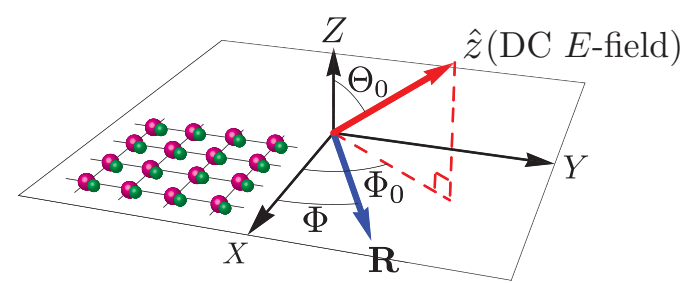

FIG. 1. (Color online) A lattice of polar molecules in the $X Y$ plane is subjected to a dc electric field along $\hat{z}$. We define the $x y z$ coordinate system as the rotation of the $X Y Z$ coordinate system around $\hat{Z}$ by $\Phi_{0}$ and then around $\hat{y}$ by $\Theta_{0}$. A vector $\mathbf{R}$ with polar coordinates $(R, \Phi)$ in the $X Y$ plane has spherical coordinates $(R, \theta, \phi)$ in the $x y z$ coordinate system.

method (DMRG) ${ }^{64}$ we compute the phase diagram of the two-legged-ladder model obtained in our polar-molecule implementation and provide evidence that-at least in this one-dimensional model-SPT phases also exist in the presence of long-range dipolar interactions.

In a major advance over Refs. 32, 33, and 65, our proposal realizes an interacting topological phase. Furthermore, relying on homogeneous microwave - not optical-dressing, our proposal is much easier to realize experimentally than the one on topological flat bands. ${ }^{65}$ Finally, since the relevant motional energy scale in our setup is the lattice band gap, our proposal can be realized at much higher motional temperature than the $t$ - $J$-type model of Refs. 32 and 33, which relies on tunneling.

Setup. We consider an array of polar molecules confined to the $X Y$ plane and pinned in a deep optical lattice with one molecule per site [see Fig. 1(a)]. Each molecule is treated as a rigid rotor with dipole moment operator $\mathbf{d}$, angular momentum operator $\mathbf{N}$, and a rotational constant $B$, and is described by the Hamiltonian $H_{0}=B \mathbf{N}^{2}-E d^{z}$ in the presence of a dc electric field $E$ along $\hat{z}$. As one turns on $E$, the simultaneous eigenstates of $\mathbf{N}^{2}$ and $N_{z}$ with eigenvalues $N(N+1)$ and $M$ adiabatically connect to eigenstates of $H_{0}$, which we denote $|N, M\rangle$. The dipole-dipole interaction between molecules $i$ and 
$j$ separated by $\mathbf{R}$ [see Fig. 1(a)] is ${ }^{66}$

$$
H_{i j}=-\frac{\sqrt{6}}{R^{3}} \sum_{q=-2}^{2}(-1)^{q} C_{-q}^{2}(\theta, \phi) T_{q}^{2}\left(\mathbf{d}_{i}, \mathbf{d}_{j}\right),
$$

where $C_{q}^{2}(\theta, \phi)=\sqrt{4 \pi / 5} \quad Y_{2, q}(\theta, \phi)$ and the many-body Hamiltonian is $H=(1 / 2) \sum_{i \neq j} H_{i j}$. Here $T_{q}^{2}\left(\mathbf{d}_{i}, \mathbf{d}_{j}\right)$ is given by $T_{ \pm 2}^{2}=d_{i}^{ \pm} d_{j}^{ \pm}, \quad T_{ \pm 1}^{2}=\left(d_{i}^{0} d_{j}^{ \pm}+d_{i}^{ \pm} d_{j}^{0}\right) / \sqrt{2}$, and $T_{0}^{2}=\left(d_{i}^{-} d_{j}^{+}+2 d_{i}^{0} d_{j}^{0}+d_{i}^{+} d_{j}^{-}\right) / \sqrt{6}$, where $d^{0}=d^{z}$ and $d^{ \pm}=\mp\left(d^{x} \pm i d^{y}\right) / \sqrt{2}$. Thus $T_{q}^{2}$ changes the total $M$ of the two molecules by $q$. For $R \sim 0.4 \mu \mathrm{m}$, the interaction energy scale is $d^{2} / R^{3} \sim 1(100) \mathrm{kHz}$ in $\mathrm{KRb}$ (LiCs).

To obtain a spin- $S$ Hamiltonian, we select in each molecule $2 S+1$ disjoint sets of $|N, M\rangle$ states and couple the states within each set to form dressed states $(\sim n$ microwave fields are needed to couple $n$ states). We then choose one ${ }^{67}$ dressed state from each set to create the spin- $S$ configuration. Projecting Eq. (1) onto the chosen spin- $S$ basis, the resulting spinspin interactions consist of five potentially independently controllable terms with angular dependences $C_{0}^{2}, \operatorname{Re}\left[C_{1}^{2}\right]$, $\operatorname{Im}\left[C_{1}^{2}\right], \operatorname{Re}\left[C_{2}^{2}\right]$, and $\operatorname{Im}\left[C_{2}^{2}\right]$. References 32 and 33 considered the special case where $S=1 / 2$, total $S^{z}$ is conserved, and only $C_{0}^{2}$ contributes. Therefore, Refs. 32 and 33 could not realize interactions featuring a direction-dependent spin anisotropy, a crucial ingredient of the present proposal. In this Rapid Communication, we evince the power of the approach beyond the special case of Refs. 32 and 33.

Interactions featuring a direction-dependent spin anisotropy. Our first demonstration of direction-dependent interactions focuses on $S=1 / 2$ and assumes that $H_{i j}$ connects a pair of molecules in the state $\left|m_{1}\right\rangle\left|m_{2}\right\rangle\left(\left|m_{1}\right\rangle\right.$ and $\left|m_{2}\right\rangle$ are dressed states) only to itself and to $\left|m_{2}\right\rangle\left|m_{1}\right\rangle$, while all the other processes are off resonant and thus negligible. Although one may be able to independently control each of the five $C_{q}^{2}$ terms, here we will focus on the $C_{0, \pm 2}^{2}$ terms since $C_{ \pm 1}^{2}$ terms are resonant only at specific values of $E .^{68}$

Consider the level configuration in Fig. 2(a). We assume the Rabi frequencies $\Omega_{ \pm}$are positive and satisfy $\left|\Omega_{ \pm}\right| \gg H_{i j}$. We take $|\uparrow\rangle=|0,0\rangle$ and $|\downarrow\rangle=\alpha|1,-1\rangle-\beta|1,1\rangle$ as our dressed spin states, where $\{\alpha, \beta\}=\left\{\Omega_{-}, \Omega_{+}\right\} / \sqrt{\Omega_{-}^{2}+\Omega_{+}^{2}}$.
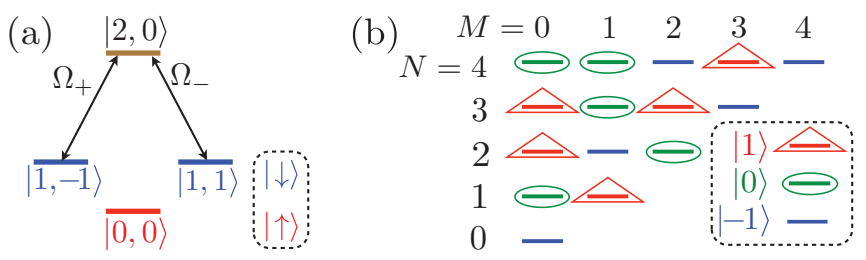

FIG. 2. (Color online) (a) The level scheme and resonant microwave coupling used to realize the Hamiltonian, Eq. (2). The dressed states we choose are $\{|\uparrow\rangle,|\downarrow\rangle\}=\left\{|0,0\rangle,\left(\Omega_{-}|1,-1\rangle-\right.\right.$ $\left.\left.\Omega_{+}|1,1\rangle\right) / \sqrt{\Omega_{-}^{2}+\Omega_{+}^{2}}\right\}$. (b) Microwave-dressed rotational levels for the SU(2)-symmetric spin-1 model. The dressed states are $|1\rangle$ (linear combination of states indicated by triangles), $|0\rangle$ (ovals), and $|-1\rangle$ (the rest). The diagram is schematic: The real system is anharmonic and levels $|N, M\rangle$ with the same $N$ are nondegenerate (unless the levels have the same $|M|)$.
Notice that $|\downarrow\rangle$ is a single-molecule eigenstate in the presence of $\Omega_{ \pm}$. The spin model is then derived by projecting $H_{i j}$ onto states $|\uparrow\rangle$ and $|\downarrow\rangle$ via the same steps as in Ref. 33 with one major difference: $d_{i}^{+} d_{j}^{+}$, featuring a $C_{-2}^{2}$ angular dependence, resonantly couples $|1,-1\rangle|0,0\rangle \rightarrow|0,0\rangle|1,1\rangle$. The result is ${ }^{69}$

$$
R^{3} H_{i j}=J_{z}(\Phi) S_{i}^{z} S_{j}^{z}+J_{x y}(\Phi)\left(S_{i}^{x} S_{j}^{x}+S_{i}^{y} S_{j}^{y}\right),
$$

where $\quad J_{z}(\Phi)=\left[1-3 \cos ^{2}\left(\Phi-\Phi_{0}\right) \sin ^{2} \Theta_{0}\right]\left(\mu_{0}-\mu_{1}\right)^{2}$, $J_{x y}(\Phi)=-\mu_{01}^{2}\left[1-3 \cos ^{2}\left(\Phi-\Phi_{0}\right) \sin ^{2} \Theta_{0}\right]+6 \alpha \beta \mu_{01}^{2}[1-$ $\left.\cos ^{2}\left(\Phi-\Phi_{0}\right)\left(1+\cos ^{2} \Theta_{0}\right)\right], \quad \mu_{0}=\left\langle 0,0\left|d^{0}\right| 0,0\right\rangle, \quad \mu_{1}=$ $\left\langle 1,1\left|d^{0}\right| 1,1\right\rangle, \quad \mu_{01}=\left\langle 1,1\left|d^{+}\right| 0,0\right\rangle$, and $\mathbf{S}_{j}$ is the spin- $1 / 2$ operator for molecule $j$. The spin anisotropy $J_{x y} / J_{z}$ of this $X X Z$ model changes depending on the polar angle $\Phi$ of the vector $\mathbf{R}$ connecting the two interacting molecules. As we discuss below, Eq. (2) allows one to study SPT phases in ladders. Another special case is a square-lattice Heisenberg model with a tunable ratio between coupling strengths on $\hat{X}$ and $\hat{Y}$ bonds. ${ }^{70}$ In the nearest-neighbor limit, this enables one to study the change from one-dimensional (uncoupled) chains to a two-dimensional behavior. Such models have also been used to explore the physics of stripes in high-temperature superconductors. ${ }^{71,72}$ While we see that even the simple level structure of Fig. 2(a) yields a wealth of exotic physics, we show below that additional features can be accessed with increased microwave control.

Degenerate dressed states and non-Abelian anyons. To realize models such as the quantum compass model, ${ }^{73}$ the Kitaev honeycomb model, ${ }^{48}$ and the Yao-Kivelson model, ${ }^{74}$ we need to go beyond Eq. (2) and realize terms, such as $S_{i}^{x} S_{j}^{x}$, that do not conserve the total $S^{z}$. To do this, we simply tune $|\uparrow\rangle$ and $|\downarrow\rangle$ to be degenerate.

As an example, consider the Kitaev honeycomb model, where interactions along $\Phi=\pi / 6, \pi / 2$, and $5 \pi / 6$ are of the form $S_{i}^{x} S_{j}^{x}, S_{i}^{y} S_{j}^{y}$, and $S_{i}^{z} S_{j}^{z}$, respectively. ${ }^{48}$ At $\left(\Theta_{0}, \Phi_{0}\right)=(0,0)$, the interaction between two molecules $i$ and $j$ is $R^{3} H_{i j}(\Phi)=\mathbf{v}(\Phi) \cdot \mathbf{M}$, where $\mathbf{v}(\Phi)=\{1,-3 \cos (2 \Phi) /$ $2,3 \sin (2 \Phi) / 2\}$ and $\mathbf{M}=\left\{\sqrt{3 / 2} T_{0}^{2}, T_{2}^{2}+T_{-2}^{2}, i T_{2}^{2}-i T_{-2}^{2}\right\}$. Since $\mathbf{v}(\pi / 6), \mathbf{v}(\pi / 2)$, and $\mathbf{v}(5 \pi / 6)$ are linearly independent, it is, in principle, possible to choose the degenerate dressed states $|\uparrow\rangle$ and $|\downarrow\rangle$ to ensure that $H_{i j}(\pi / 6) \propto S_{i}^{x} S_{j}^{x}$, $H_{i j}(\pi / 2) \propto S_{i}^{y} S_{j}^{y}$, and $H_{i j}(5 \pi / 6) \propto S_{i}^{z} S_{j}^{z}$. In Ref. 75, we show that, with $\sim 25$ microwave fields, such a choice of dressed states is indeed possible, allowing one to realize the Kitaev $B$ phase in the presence of a magnetic field. This gapped phase supports non-Abelian anyonic excitations, which can be used, for example, for topologically protected quantum state transfer ${ }^{76}$ and quantum computing. ${ }^{48}$

$S>1 / 2$ and the bilinear-biquadratic model. We now show that one can extend this tremendous control over spin-spin interactions to $S>1 / 2$. In particular, we show how to obtain the general SU(2)-symmetric spin1 Hamiltonian, i.e., the bilinear-biquadratic Hamiltonian $\cos (\gamma) \mathbf{S}_{i} \cdot \mathbf{S}_{j}+\sin (\gamma)\left(\mathbf{S}_{i} \cdot \mathbf{S}_{j}\right)^{2}$, which has a rich phase diagram even in one dimension. ${ }^{17,47,77}$ In particular, $\gamma=\pi / 4$ and $\arctan (1 / 3)$ give the SU(3)-symmetric and the AffleckLieb-Kennedy-Tasaki (AKLT) ${ }^{78}$ Hamiltonians, respectively. One can also consider generalizations to $\mathrm{SU}(N)$ with arbitrary $N$ as well as away from $\mathrm{SU}(2){ }^{79}$ 
(a)
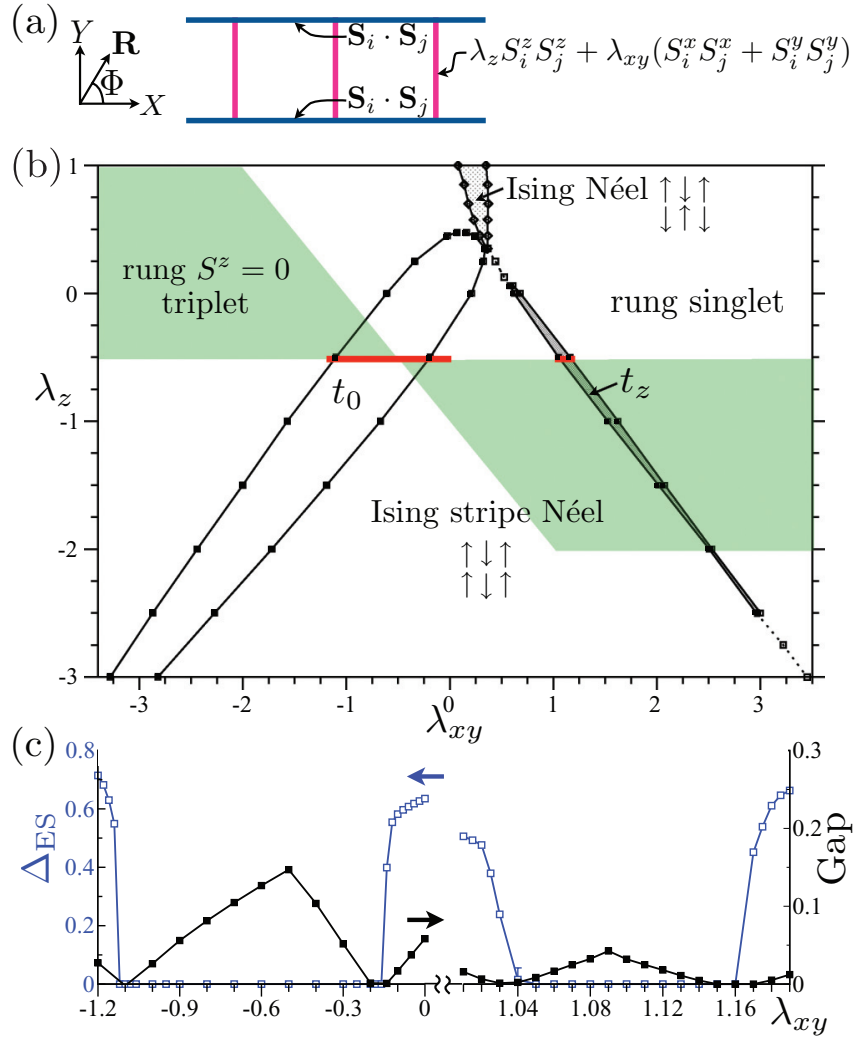

FIG. 3. (Color online) Spin ladder of Ref. 50 and its phase diagram showing SPT phases in the presence of long-range interactions. (a) The nearest-neighbor model. (b) Phase diagram in the presence of long-range interactions. The shaded area indicates points achievable with the simple configuration of Fig. 2(a). The shaded area does not extend past the limits of the vertical axis, while it extends infinitely far along the horizontal axis. (c) Entanglement splitting $\Delta_{\mathrm{ES}}$ (open boxes, left axis) and energy gap (solid boxes, right axis) of the SPT phases along $\lambda_{z}=-0.5$ cuts (bold, red lines) shown in (b).

We build our spin-1 dressed-state basis $\{|1\rangle,|0\rangle,|-1\rangle\}$ from the 15 bare levels shown in Fig. 2(b). For simplicity, the model presented here will have only the $C_{0}^{2}$ term. We work at $E=3.244 B / d[=13(7) \mathrm{kV} / \mathrm{cm}$ in $\mathrm{KRb}(\mathrm{LiCs})]$, for which the bare-states process $|1,0\rangle|1,0\rangle \rightarrow|2,0\rangle|0,0\rangle$ is resonant. Furthermore, we choose the energies of the dressed states to make the process $|0\rangle|0\rangle \rightarrow|-1\rangle|1\rangle$ resonant. ${ }^{80}$ The latter is needed to engineer the $S_{i}^{-} S_{j}^{+}$term present in the desired Hamiltonian. Aside from this exception, we again assume that a pair of molecules in dressed states $\left|m_{1}\right\rangle\left|m_{2}\right\rangle$ is connected via $H_{i j}$ only to itself and to $\left|m_{2}\right\rangle\left|m_{1}\right\rangle$. Using 12 microwaves to create the dressed states in Fig. 2(b), we find ${ }^{69}$ that we can achieve any $\gamma$ and, thus, any bilinear-biquadratic Hamiltonian. Removing all five $N=4$ states, we are left with just eight microwaves, which simplifies the experimental implementation but at the cost of only accessing the $\gamma=1.1$ point. The typical strength of interactions achieved is $R^{3} H_{i j} \sim 0.01 d^{2}$. Stronger interactions and a reduced number of microwaves might be achievable by further optimizing the choice of levels and microwaves.

SPT phases in spin ladders. We now turn to the focus of our work: We use Eq. (2) to implement a specific ladder model [see Fig. 3(a)] introduced in Ref. 50 and shown to support nontrivial SPT phases for nearest-neighbor interactions. The symmetries protecting these interacting topological phases are the exchange $\sigma$ of the two legs and $D_{2}=\left\{E, R_{x}, R_{y}, R_{z}\right\}$, where $E$ is the identity and $R_{\alpha}$ is a $\pi$ pulse around the axis $\alpha$ on all spins. Note that although we focus on a ladder system since it is amenable to a numerically exact treatment, we expect an even richer phase diagram in dimensions $D>1$.

While for our choice of levels $b \equiv \mu_{01}^{2} /\left(\mu_{0}-\mu_{1}\right)^{2}$ satisfies $b \in[2.6, \infty)$, any $b \geqslant 0$ can be accessed by using reduced nuclear spin overlaps between $|\uparrow\rangle$ and $|\downarrow\rangle$ (see experimental considerations below) or by choosing $|N, M\rangle$ with different $N$. To ensure the $\sigma$ symmetry, we take $\Phi_{0}=\pi / 2$. To ensure a Heisenberg Hamiltonian along $\Phi=0$, we choose $\alpha \beta=(b+1) /(6 b)$. Since $\alpha \beta \in[0,1 / 2]$ and $b \geqslant 0, b$ can go from $1 / 2$ to $\infty$. Rescaling the interaction by $\left(\mu_{0}-\mu_{1}\right)^{2}$ (which goes up to $\approx 0.1 d^{2}$ for our choice of levels), defining $\lambda_{z}=1-3 \cos ^{2} \Theta_{0}$ (tunable via $\Theta_{0}$ between -2 and 1 ), and $\lambda_{x y}=-\frac{2(b+1)}{3}-\frac{4 b+1}{3} \lambda_{z}$ [see shaded area in Fig. 3(b)], we obtain Eq. (2) with $J_{z}(\Phi)=1-\left(1-\lambda_{z}\right) \sin ^{2} \Phi$ and $J_{x y}(\Phi)=1-\left(1-\lambda_{x y}\right) \sin ^{2} \Phi$. As desired, at the nearestneighbor level, these expressions for $J_{z}(\Phi)$ and $J_{x y}(\Phi)$ reproduce Fig. 3(a).

In our implementation using polar molecules, the nearestneighbor interactions are replaced by dipolar interactions, which give rise to nontrivial longer-range corrections. In order to investigate the role of these corrections, we numerically calculate the phase diagram of the spin ladder with long-range interactions, as shown in Fig. 3(b), using DMRG on 200 rungs with smooth boundary conditions. ${ }^{81}$ By performing a finite-size scaling using systems with up to 400 rungs, we estimate the finite-size effects to be comparable to the size of the symbols in Fig. 3(b). The phase diagram is qualitatively similar to the nearest-neighbor case ${ }^{50}$ and exhibits the same six phases, including the two SPT phases. In Ref. 50's language, the four nontopological phases are the Ising Néel, the Ising stripe Néel, and two product phases of rung singlets and $S^{z}=0$ rung triplets. The remaining two phases, $t_{0}$ and $t_{z}$, are two out of seven nontrivial SPT phases protected by $D_{2} \times \sigma .{ }^{50}$ The $t_{0}$ phase can be connected to the Haldane phase ${ }^{82}$ and to the AKLT state ${ }^{78}$ by treating the triplet states on each rung as a spin-1 particle. Meanwhile, in the nearest-neighbor case, $t_{z}$ is obtained from $t_{0}$ by taking $|\uparrow\rangle \rightarrow-|\uparrow\rangle$ on one of the legs, which is the $\lambda_{x y} \rightarrow-\lambda_{x y}$ symmetry of the nearest-neighbor phase diagram. ${ }^{50}$ Long-range interactions break this symmetry and, in particular, reduce the size of the $t_{z}$ phase relative to the $t_{0}$ phase as a result of substantial next-nearest-neighbor $S_{i}^{x} S_{j}^{x}+S_{i}^{y} S_{j}^{y}$ interactions for $\lambda_{x y}>0$.

We observe that the $t_{z}$ phase is sensitive to artificial cutoffs in the interaction range, so we use matrix product operators within DMRG to provide an efficient description of interactions without a cutoff; instead, we fit the long-range interactions to a sum of exponentials. ${ }^{69,83-86}$ The boundaries of the Ising Néel and Ising stripe Néel phases were obtained by calculating the corresponding order parameters. We identified the two rung phases by calculating $\left\langle S_{i}^{x} S_{j}^{x}+S_{i}^{y} S_{j}^{y}\right\rangle$ on the rungs and by verifying that the gap does not close as $\left|\lambda_{x y}\right|$ increases to large values where the system is ultimately exactly solvable. The boundaries of the SPT phases were obtained by computing the entanglement splitting, which we define as $\Delta_{\mathrm{ES}}=\sum_{j=\text { odd }}\left(w_{j}-w_{j+1}\right)$, where $w_{j}$ are the eigenvalues of the reduced density matrix for a bipartition at the center 
of the system, sorted from largest to smallest. Due to their twofold degenerate entanglement spectrum, ${ }^{63}$ SPT phases have $\Delta_{\mathrm{ES}}=0$, as shown in Fig. 3(c). We have also verified that all phases are gapped. Interestingly, the energy gap in the SPT phases, shown in Fig. 3(c), exhibits a cusp indicative of a level crossing, which deserves further investigation. Finally, using again systems with 200 rungs, we added to the Hamiltonian a small term $\propto S_{i}^{x} \pm S_{j}^{x}$, where $i$ and $j$ are sites on the same edge rung. Referring to operators that split the edge degeneracy as active operators, we verified the prediction ${ }^{50}$ that $S_{i}^{x}+S_{j}^{x}$ is an active operator for the $t_{0}$ phase while $S_{i}^{x}-S_{j}^{x}$ is not, and vice versa for the $t_{z}$ phase.

Experimental considerations. By temporarily breaking the $D_{2} \times \sigma$ symmetry, the phases $t_{0}$ and $t_{z}$ can be prepared from rung phases without closing the gap. ${ }^{59}$ Turning to the question of detection, an SPT phase can be classified ${ }^{50}$ by finding its active operators, i.e., those operators that split the edge degeneracy. We propose to diagnose this splitting by measuring an active operator in linear response to the application of that same operator at frequency $\omega$ and looking for the zero-bias $(\omega=0)$ peak. Repeating the same procedure for inactive operators will yield no zero-bias peak. In our implementation, a $z$ magnetic field proportional to $\mu_{0}^{2}-\mu_{1}^{2}$ naturally arises at the edges from dipole-dipole interactions. ${ }^{69}$ Since such a field constitutes an active operator of the $t_{0}$ and $t_{z}$ phases, it is natural to probe the response of the system by tuning $\mu_{0}^{2}-\mu_{1}^{2}$ with the dc electric field. In combination with a spectroscopic verification of the bulk gap, the response to active operators allows one to detect and classify SPT phases. A more modest first experimental step could be to use a Ramsey-type experiment ${ }^{41}$ to benchmark how accurately the molecules emulate the desired Hamiltonians.

Polar alkali-metal dimers have a hyperfine structure $H_{\mathrm{hf}},{ }^{33,87}$ which we have ignored so far. We will illustrate how to deal with $H_{\mathrm{hf}}$ for the specific case of $S=1 / 2$. Assuming that microwave Rabi frequencies $\Omega_{i}$ are much larger than $H_{\mathrm{hf}}$, we can project the hyperfine structure on dressed states $|\uparrow\rangle$ and $|\downarrow\rangle$. The necessary conditions $H_{\mathrm{hf}} \ll \Omega_{i} \ll B$ are easy to satisfy: For example, in ${ }^{40} \mathrm{~K}^{87} \mathrm{Rb}, H_{\mathrm{hf}} \sim(2 \pi) 1 \mathrm{MHz}$ and the rotational constant is $B \sim(2 \pi) 1 \mathrm{GHz}$. The simplest situation arises when an applied magnetic field —of a strength already experimentally used ${ }^{1}$-makes $\left\langle\uparrow\left|H_{\mathrm{hf}}\right| \uparrow\right\rangle$ and $\left\langle\downarrow\left|H_{\mathrm{hf}}\right| \downarrow\right\rangle$ diagonal in the same basis of decoupled nuclear spins. The nuclear-spin degree of freedom can then be eliminated by working with a single state from this basis. For smaller magnetic fields, one could prepare the system in any pair of nonorthogonal eigenstates of $\left\langle\uparrow\left|H_{\mathrm{hf}}\right| \uparrow\right\rangle$ and $\left\langle\downarrow\left|H_{\mathrm{hf}}\right| \downarrow\right\rangle$. An imperfect overlap of these two states will effectively reduce the transition dipole moment between $|\uparrow\rangle$ and $|\downarrow\rangle$, resulting in an additional control knob of the interactions.

Controlling tens of independent microwave frequencies in the frequency range required by our proposal is straightforward. ${ }^{88}$ The two uncertainties involved are in the generation of the microwaves and in the coupling to molecules. The latter is dominant: Current ultracold molecule experiments observe only $0.1 \%$ deviations in their $\sim 1 \mathrm{~ms}$ microwave pulses without any particular optimization, ${ }^{89}$ and this is expected to be independent of the number of microwaves applied. Polarization control is more challenging. However, this should be attainable, for example, by simply interfering the outputs of two independently controlled microwave horns.

Outlook. While dipolar interactions did not destroy the SPT phases in our example, quantum magnets with long-range interactions have recently been shown to harbor unusual and often dimension-specific physics. ${ }^{90-96}$ The polar-molecule experiment we propose could therefore help guide the theoretical understanding of these effects in two-dimensional and threedimensional systems, including SPT phases, where efficient numerical methods are lacking. In fact, the classification of SPT phases is yet to be extended to models with long-range interactions. Finally, we expect our methods to be immediately extendable to other dipole-dipole interacting systems such as Rydberg atoms, ${ }^{4,5}$ magnetic atoms, ${ }^{6,7}$ and magnetic defects in solids. $^{8-10}$

We thank J. Preskill, J. Ye, D. Jin, M. Lukin, N. Yao, S. Michalakis, A. Turner, N. Schuch, N. Lindner, G. Evenbly, M. Baranov, J. Taylor, S. Stellmer, W. Campbell, M. Foss-Feig, M. Hermele, V. Gurarie, X.-G. Wen, Z.-X. Liu, and M. Oshikawa for discussions. This work was supported by NSF, IQIM, NRC, AFOSR, ARO, the ARO-DARPA-OLE program, and the Lee A. DuBridge and Gordon and Betty Moore foundations. S.R.M. and K.R.A.H. thank KITP for hospitality. We acknowledge the use of the Janus supercomputer facilities at CU Boulder. This manuscript is the contribution of NIST and is not subject to U.S. copyright.

${ }^{1}$ K. K. Ni et al., Science 322, 231 (2008).

${ }^{2}$ K. Aikawa, D. Akamatsu, M. Hayashi, K. Oasa, J. Kobayashi, P. Naidon, T. Kishimoto, M. Ueda, and S. Inouye, Phys. Rev. Lett. 105, 203001 (2010).

${ }^{3}$ J. Deiglmayr, A. Grochola, M. Repp, K. Mörtlbauer, C. Glück, J. Lange, O. Dulieu, R. Wester, and M. Weidemüller, Phys. Rev. Lett. 101, 133004 (2008).

${ }^{4}$ M. Saffman, T. G. Walker, and K. Mølmer, Rev. Mod. Phys. 82, 2313 (2010).

${ }^{5}$ P. Schauß, M. Cheneau, M. Endres, T. Fukuhara, S. Hild, A. Omran, T. Pohl, C. Gross, S. Kuhr, and I. Bloch, Nature (London) 491, 87 (2012).

${ }^{6}$ K. Aikawa, A. Frisch, M. Mark, S. Baier, A. Rietzler, R. Grimm, and F. Ferlaino, Phys. Rev. Lett. 108, 210401 (2012).

${ }^{7}$ M. Lu, N. Q. Burdick, and B. L. Lev, Phys. Rev. Lett. 108, 215301 (2012).

${ }^{8}$ L. Childress, M. V. Gurudev Dutt, J. M. Taylor, A. S. Zibrov, F. Jelezko, J. Wrachtrup, P. R. Hemmer, and M. D. Lukin, Science 314, 281 (2006).

${ }^{9}$ G. Balasubramanian et al., Nat. Mater. 8, 383 (2009).

${ }^{10}$ J. R. Weber, W. F. Koehl, J. B. Varley, A. Janotti, B. B. Buckley, C. G. Van de Walle, and D. D. Awschalom, Proc. Natl. Acad. Sci. (USA) 107, 8513 (2010).

${ }^{11}$ M. A. Baranov, Phys. Rep. 464, 71 (2008).

${ }^{12}$ T. Lahaye, C. Menotti, L. Santos, M. Lewenstein, and T. Pfau, Rep. Prog. Phys. 72, 126401 (2009).

${ }^{13}$ C. Trefzger, C. Menotti, B. Capogrosso-Sansone, and M. Lewenstein, J. Phys. B 44, 193001 (2011). 
${ }^{14}$ M. A. Baranov, M. Dalmonte, G. Pupillo, and P. Zoller, Chem. Rev. 112, 5012 (2012).

${ }^{15}$ R. Barnett, D. Petrov, M. Lukin, and E. Demler, Phys. Rev. Lett. 96, 190401 (2006).

${ }^{16}$ A. Micheli, G. K. Brennen, and P. Zoller, Nat. Phys. 2, 341 (2006).

${ }^{17}$ G. K. Brennen, A. Micheli, and P. Zoller, New J. Phys. 9, 138 (2007).

${ }^{18}$ H. P. Büchler, A. Micheli, and P. Zoller, Nat. Phys. 3, 726 (2007).

${ }^{19}$ A. V. Gorshkov, P. Rabl, G. Pupillo, A. Micheli, P. Zoller, M. D. Lukin, and H. P. Buchler, Phys. Rev. Lett. 101, 073201 (2008).

${ }^{20}$ M. L. Wall and L. D. Carr, New J. Phys. 11, 055027 (2009).

${ }^{21}$ M. L. Wall and L. D. Carr, Phys. Rev. A 82, 013611 (2010).

${ }^{22}$ J. Schachenmayer, I. Lesanovsky, A. Micheli, and A. J. Daley, New J. Phys. 12, 103044 (2010).

${ }^{23}$ J. Pérez-Ríos, F. Herrera, and R. V. Krems, New J. Phys. 12, 103007 (2010).

${ }^{24}$ F. Herrera, M. Litinskaya, and R. V. Krems, Phys. Rev. A 82, 033428 (2010).

${ }^{25}$ H. Weimer, M. Muller, I. Lesanovsky, P. Zoller, and H. P. Buchler, Nat. Phys. 6, 382 (2010).

${ }^{26}$ M. Dalmonte, G. Pupillo, and P. Zoller, Phys. Rev. Lett. 105, 140401 (2010).

${ }^{27}$ T. Pohl, E. Demler, and M. D. Lukin, Phys. Rev. Lett. 104, 043002 (2010).

${ }^{28}$ S. Ospelkaus, K.-K. Ni, G. Quéméner, B. Neyenhuis, D. Wang, M. H. G. de Miranda, J. L. Bohn, J. Ye, and D. S. Jin, Phys. Rev. Lett. 104, 030402 (2010).

${ }^{29}$ M. H. G. de Miranda et al., Nat. Phys. 7, 502 (2011).

${ }^{30}$ J. P. Kestner, B. Wang, J. D. Sau, and S. Das Sarma, Phys. Rev. B 83, 174409 (2011).

${ }^{31}$ M. Lemeshko, Phys. Rev. A 83, 051402 (2011).

${ }^{32}$ A. V. Gorshkov, S. R. Manmana, G. Chen, J. Ye, E. Demler, M. D. Lukin, and A. M. Rey, Phys. Rev. Lett. 107, 115301 (2011).

${ }^{33}$ A. V. Gorshkov, S. R. Manmana, G. Chen, E. Demler, M. D. Lukin, and A. M. Rey, Phys. Rev. A 84, 033619 (2011).

${ }^{34}$ L. Mathey, K. J. Günter, J. Dalibard, and A. Polkovnikov, arXiv:1112.1204.

${ }^{35}$ Y. L. Zhou, M. Ortner, and P. Rabl, Phys. Rev. A 84, 052332 (2011).

${ }^{36}$ M. Dalmonte, P. Zoller, and G. Pupillo, Phys. Rev. Lett. 107, 163202 (2011).

${ }^{37}$ M. Babadi and E. Demler, Phys. Rev. A 84, 033636 (2011).

${ }^{38}$ Q. Wei, S. Kais, B. Friedrich, and D. Herschbach, J. Chem. Phys. 134, 124107 (2011).

${ }^{39}$ A. Chotia, B. Neyenhuis, S. A. Moses, B. Yan, J. P. Covey, M. Foss-Feig, A. M. Rey, D. S. Jin, and J. Ye, Phys. Rev. Lett. 108, 080405 (2012).

${ }^{40}$ H. Weimer, N. Y. Yao, C. R. Laumann, and M. D. Lukin, Phys. Rev. Lett. 108, 100501 (2012).

${ }^{41}$ K. R. A. Hazzard, S. R. Manmana, M. Foss-Feig, and A. M. Rey, Phys. Rev. Lett. 110, 075301 (2013).

${ }^{42}$ M. Lemeshko, R. V. Krems, and H. Weimer, Phys. Rev. Lett. 109, 035301 (2012).

${ }^{43}$ T. Sowiński, O. Dutta, P. Hauke, L. Tagliacozzo, and M. Lewenstein, Phys. Rev. Lett. 108, 115301 (2012).

${ }^{44}$ M. Maik, P. Hauke, O. Dutta, J. Zakrzewski, and M. Lewenstein, New J. Phys. 14, 113006 (2012).

${ }^{45}$ M. L. Wall, E. Bekaroglu, and L. D. Carr, arXiv:1212.3042.

${ }^{46}$ J. Zhu, S. Kais, Q. Wei, D. Herschbach, and B. Friedrich, J. Chem. Phys. 138, 024104 (2013).
${ }^{47}$ U. Schollwöck, T. Jolicoeur, and T. Garel, Phys. Rev. B 53, 3304 (1996).

${ }^{48}$ A. Kitaev, Ann. Phys. 321, 2 (2006).

${ }^{49}$ I. Bloch, J. Dalibard, and W. Zwerger, Rev. Mod. Phys. 80, 885 (2008).

${ }^{50}$ Z.-X. Liu, Z.-B. Yang, Y.-J. Han, W. Yi, and X.-G. Wen, Phys. Rev. B 86, 195122 (2012).

${ }^{51}$ A. P. Schnyder, S. Ryu, A. Furusaki, and A. W. W. Ludwig, Phys. Rev. B 78, 195125 (2008).

${ }^{52}$ A. P. Schnyder, S. Ryu, A. Furusaki, and A. W. W. Ludwig, in Advances in Theoretical Physics: Landau Memorial Conference, edited by V. Lebedev and M. Feigel'man, AIP Conf. Proc. Vol. 1134 (AIP, Melville, NY, 2009), p. 10.

${ }^{53}$ A. Kitaev, in Ref. 52, p. 22.

${ }^{54}$ S. Ryu, A. P. Schnyder, A. Furusaki, and A. W. W. Ludwig, New J. Phys. 12, 065010 (2010).

${ }^{55}$ Z.-C. Gu and X.-G. Wen, Phys. Rev. B 80, 155131 (2009).

${ }^{56}$ X. Chen, Z.-C. Gu, and X.-G. Wen, Phys. Rev. B 83, 035107 (2011).

${ }^{57}$ X. Chen, Z.-C. Gu, Z.-X. Liu, and X.-G. Wen, arXiv:1106.4772.

${ }^{58}$ F. Pollmann, E. Berg, A. M. Turner, and M. Oshikawa, Phys. Rev. B 85, 075125 (2012).

${ }^{59}$ N. Schuch, D. Pérez-García, and I. Cirac, Phys. Rev. B 84, 165139 (2011).

${ }^{60}$ J. Alicea, Rep. Prog. Phys. 75, 076501 (2012).

${ }^{61}$ E. G. Dalla Torre, E. Berg, and E. Altman, Phys. Rev. Lett. 97, 260401 (2006).

${ }^{62}$ M. Endres et al., Science 334, 200 (2011).

${ }^{63}$ F. Pollmann, A. M. Turner, E. Berg, and M. Oshikawa, Phys. Rev. B 81, 064439 (2010).

${ }^{64}$ U. Schollwöck, Rev. Mod. Phys. 77, 259 (2005).

${ }^{65}$ N. Y. Yao, C. R. Laumann, A. V. Gorshkov, S. D. Bennett, E. Demler, P. Zoller, and M. D. Lukin, Phys. Rev. Lett. 109, 266804 (2012).

${ }^{66}$ J. M. Brown and A. Carrington, Rotational Spectroscopy of Diatomic Molecules (Cambridge University Press, Cambridge, UK, 2003).

${ }^{67}$ One could also choose fewer sets and choose more than one dressed state from the same set.

${ }^{68}$ For example, at $E \approx 4 B / d,|1,0\rangle|1,1\rangle$ and $|0,0\rangle|2,2\rangle$ are degenerate.

${ }^{69}$ See Supplemental Material at http://link.aps.org/supplemental/ 10.1103/PhysRevB.87.081106 for details.

${ }^{70}$ Y. J. Kim and R. J. Birgeneau, Phys. Rev. B 62, 6378 (2000).

${ }^{71}$ A. H. Castro Neto and D. Hone, Phys. Rev. Lett. 76, 2165 (1996).

${ }^{72}$ C. N. A. van Duin and J. Zaanen, Phys. Rev. Lett. 80, 1513 (1998).

${ }^{73}$ K. I. Kugel and D. I. Khomskii, Sov. Phys. JETP 37, 725 (1973).

${ }^{74}$ H. Yao and S. A. Kivelson, Phys. Rev. Lett. 99, 247203 (2007).

${ }^{75}$ A. V. Gorshkov, K. R. A. Hazzard, and A. M. Rey, arXiv:1301.5636.

${ }^{76}$ N. Y. Yao, C. R. Laumann, A. V. Gorshkov, H. Weimer, L. Jiang, J. I. Cirac, P. Zoller, and M. D. Lukin, arXiv:1110.3788.

${ }^{77}$ J. J. Garcia-Ripoll, M. A. Martin-Delgado, and J. I. Cirac, Phys. Rev. Lett. 93, 250405 (2004).

${ }^{78}$ I. Affleck, T. Kennedy, E. H. Lieb, and H. Tasaki, Phys. Rev. Lett. 59, 799 (1987).

${ }^{79}$ E. Berg, E. G. Dalla Torre, T. Giamarchi, and E. Altman, Phys. Rev. B 77, 245119 (2008). 
${ }^{80} \mathrm{~A}$ different choice can controllably introduce a $\sum_{i}\left(S_{i}^{z}\right)^{2}$ term, which can be useful in certain cases (Ref. 79).

${ }^{81}$ M. Vekić and S. R. White, Phys. Rev. Lett. 71, 4283 (1993).

${ }^{82}$ F. D. M. Haldane, Phys. Rev. Lett. 50, 1153 (1983).

${ }^{83}$ I. P. McCulloch, arXiv:0804.2509.

${ }^{84}$ G. M. Crosswhite, A. C. Doherty, and G. Vidal, Phys. Rev. B 78, 035116 (2008).

${ }^{85}$ B. Pirvu, V. Murg, J. I. Cirac, and F. Verstraete, New J. Phys. 12, 025012 (2010).

${ }^{86}$ E. M. Stoudenmire and S. R. White, New J. Phys. 12, 055026 (2010).

${ }^{87}$ J. Aldegunde, B. A. Rivington, P. S. Zuchowski, and J. M. Hutson, Phys. Rev. A 78, 033434 (2008).

${ }^{88}$ B. C. Dian, G. G. Brown, K. O. Douglass, and B. H. Pate, Science 320, 924 (2008).
${ }^{89} \mathrm{~J}$. Ye (private communication).

${ }^{90}$ X. L. Deng, D. Porras, and J. I. Cirac, Phys. Rev. A 72, 063407 (2005).

${ }^{91}$ P. Hauke, F. M. Cucchietti, A. Muller-Hermes, M.-C. Banuls, J. I. Cirac, and M. Lewenstein, New J. Phys. 12, 113037 (2010).

${ }^{92}$ D. Peter, S. Müller, S. Wessel, and H. P. Büchler, Phys. Rev. Lett. 109, 025303 (2012).

${ }^{93}$ T. Koffel, M. Lewenstein, and L. Tagliacozzo, Phys. Rev. Lett. 109, 267203 (2012).

${ }^{94}$ M. L. Wall and L. D. Carr, New J. Phys. 14, 125015 (2012).

${ }^{95}$ V. Nebendahl and W. Dür, Phys. Rev. B 87, 075413 (2013).

${ }^{96}$ A. Cadarso, M. Sanz, M. M. Wolf, J. I. Cirac, and D. Perez-Garcia, Phys. Rev. B 87, 035114 (2013). 